\title{
ТЕПЛОЭНЕРГЕТИКА
}

\section{УДК 662.61}

\section{МЕТОДИКА ОПТИМИЗАЦИИ ПОВЕРХНОСТИ ВСТРОЕННОГО РЕКУПЕРАТОРА РЕКУПЕРАТИВНОЙ ГОРЕЛКИ ПО КРИТЕРИЮ МАКСИМУМА ЭНЕРГЕТИЧЕСКОГО КОЭФФИЦИЕНТА}

\author{
А.Б. БИРЮКОВ, А.Н. ЛЕБЕДЕВ, П.А. ГНИТИЁВ, Я.С. ВЛАСОВ \\ ГОУВПО «Донецкий национальный технический университет», г. Донецк, Украина \\ E-mail: birukov.ttf@gmail.com, gnitiev.pavel@gmail.com, lan@donntu.org
}

\begin{abstract}
Авторское резюме
Состояние вопроса. Современным направлением сокращения расхода топлива при тепловой обработке металла в печах является использование рекуперативных горелок. Использование таких устройств позволяет существенно сократить расход топлива. Несмотря на объективные теплотехнические преимущества, распространение такого оборудования сдерживается его высокой стоимостью. Ведутся исследования по определению рационального профриля ребер и оптимизации массогабаритных характеристик встроенного рекуператора в целом. Имеются результаты, позволяющие при прочих равных условиях уменьшить либо массу рекуператора, либо его аэродинамическое сопротивление. Однако, поскольку такие изменения входят в противоречие друг с другом, необходима компромиссная постановка задачи. В настоящее время отсутствует набор общепринятых методик теплотехнического расчета рекуперативных горелок. В связи с этим актуальным является создание методики оптимизации поверхности встроенного рекуператора по критерию максимизации энергетического коэфффициента.

Материалы и методы. Для проведения исследования использованы элементы теории рекуперативных теплообменников для противоточного течения сред и ранее апробированная методика определения температур нагретого воздуха и охлажденных продуктов сгорания после прохождения рекуператора. Также для исследования использована известная концепция энергетического коэффрициента.

Результаты. Разработана методика оптимизации поверхности встроенного рекуператора по критерию максимизации энергетического коэффициента, сущность которой заключается в составлении выражения для определения энергетического коэффициента, приравнивании производной этой величины нулю и численном разрешении полученного уравнения относительно поверхности теплообмена. Использование разработанной методики проиллюстрировано на примере рекуперативной горелки с тепловой мощностью 500 кВт. Для диапазона изменения коэффициента теплопередачи, отнесенного к гладкотрубной поверхности, от 75 до $200 \mathrm{BT} /\left(\mathrm{M}^{2} \cdot \mathrm{K}\right)$ зависимость оптимального значения гладкотрубной части поверхности теплообмена встроенного рекуператора от коэффициента теплопередачи установлена в виде полинома пятой степени.

Выводы. Разработанная методика важна для проектирования рекуперативных горелок, повышения их эффективности и снижения стоимости их производства в отечественных условиях. Погрешность методики, связанная с допущением о постоянстве коэффициента теплопередачи при изменении длины встроенного рекуператора, не превышает $5 \%$.
\end{abstract}

Ключевые слова: рекуперативная горелка, встроенный рекуператор, поверхность теплообмена, энергетический коэфффициент 


\title{
OPTIMIZATION OF THE BUILT-IN RECUPERATOR SURFACE OF RECUPERATIVE BURNER ACCORDING TO THE CRITERION OF ENERGY COEFFICIENT MAXIMUM
}

\author{
A.B. BIRYUKOV, A.N. LEBEDEV, P.A. GNITIEV, Ya.S. VLASOV \\ Donetsk National Technical University, Donetsk, Ukraine \\ E-mail: birukov.ttf@gmail.com, gnitiev.pavel@gmail.com, lan@donntu.org
}

\begin{abstract}
Background. The recuperative burners is a modern direction of reducing fuel consumption during the heat treatment of metals in furnaces. Their use can significantly reduce fuel consumption. Despite the evident advantages, the spread of such equipment is constrained due to its high cost. The research is conducted for determining ribs rational profile and optimizing mass and size characteristics of a built-in recuperator. There are results that make it possible to reduce either the recuperator mass or its aerodynamic resistance. However, such changes contradict each other, so a compromise solution must be found. Currently, there are no generally accepted methods of thermotechnical calculations for recuperative burners. This work aims to develop a methodology to optimize the built-in recuperator surface according to the criterion of maximizing the energy coefficient.

Materials and methods. To conduct the study, the elements of recuperative heat exchange theory for counter-flow media were used. The proven methodology for determining the temperature of heated air and cooled combustion products after recuperator was applied. Also, the known concept of energy coefficient was used for the research.

Results. A technique has been developed to optimize the surface of the incorporated heat exchanger according to the criterion of maximizing the energy coefficient. The technique includes composing an expression for determining the energy coefficient, taking its derivative and equating it to zero with further solving the obtained equation with respect to heat exchange surface. The developed method was used in the recuperative burner with the thermal power of $500 \mathrm{~kW}$. For the range of heat transfer coefficient $75-200 \mathrm{~W} /\left(\mathrm{m}^{2} \cdot \mathrm{K}\right)$ associated to the smoothtube part, a fifth-degree polynomial has been determined which describes the dependence of the smoothtube part optimal value of a built-in recuperator surface on the heat transfer coefficient.

Conclusions. The developed technique is important for recuperative burners design, for increasing their efficiency and reducing their production cost. The methodology error associated with the assumption that the heat transfer coefficient is constant when the length of the built-in recuperator changes does not exceed $5 \%$.
\end{abstract}

Key words: recuperative burner, built-in recuperator, heat exchange surface, energy coefficient

DOI: $10.17588 / 2072-2672.2020 .1 .005-011$

Введение. Повышение энергоэффективности технологических процессов является одной из приоритетных задач в любой отрасли промышленности. При реализации технологий тепловой обработки материалов в печах необходимо достигать сокращения величины удельного расхода топлива. Одним из основных направлений работы в этом направлении является использование теплоты уходящих продуктов сгорания для подогрева компонентов горения. Повышение величины коэффициента рекуперации позволяет увеличить коэффициент использования топлива и КПД печного агрегата, а значит, достичь сокращения удельного расхода топлива [1]. Одним из способов существенного повышения коэффициента рекуперации является использование рекуперативных горелок [2-4]. С их помощью удается нагреть основной поток воздуха, идущего на горение, до температур порядка $800{ }^{\circ} \mathrm{C}$, что позволяет достичь существенного сокращения удельного расхода топлива по сравнению с системами на базе центрального рекуператора. Однако до сих пор рекуперативные горелки не получили широкого распространения ни в отечественной, ни в мировой практике по причине своей высокой стоимости и длительного срока окупаемости средств, вложенных в приобретение отопительных систем на базе рекуперативных горелок [2].

Поиск путей совершенствования конструкций рекуперативных горелок является важной научно-технической задачей, решение которой позволит сократить стоимость этого оборудования и сделать целесообразным его использование при создании новых печных агрегатов и реконструкции существующих [2-4]. Схематичное изображение конструктивных особенностей горелки представлено на рис. 1. 


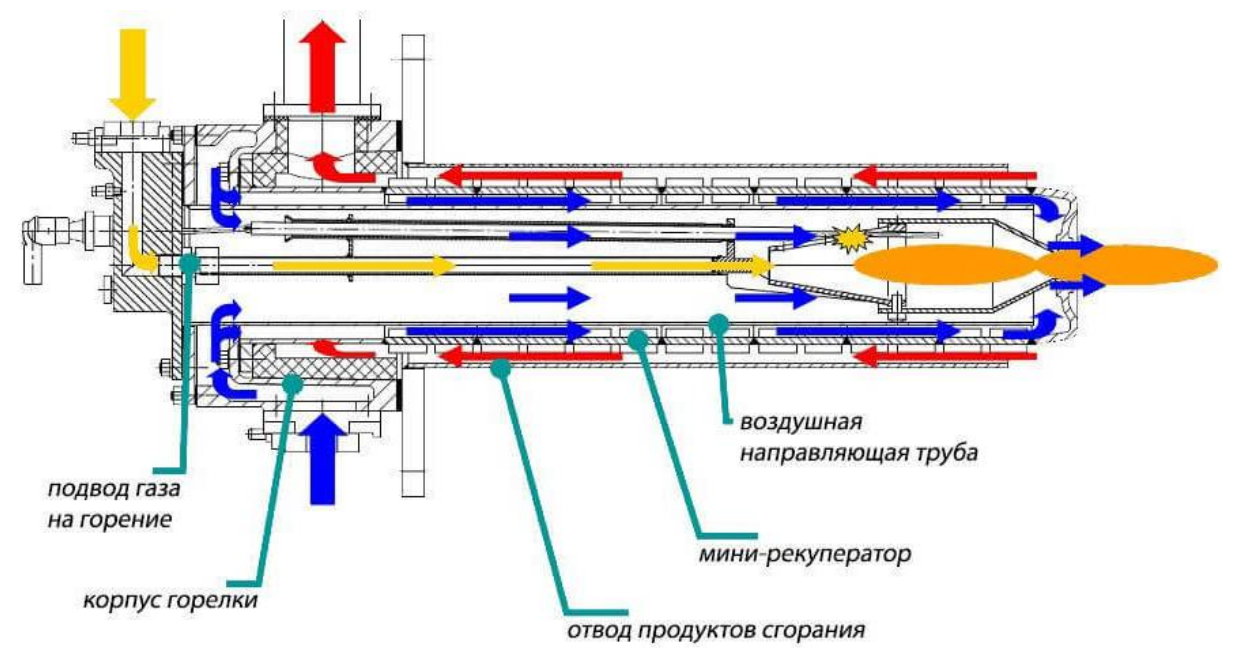

Рис. 1. Устройство рекуперативной горелки

Одной из основных характеристик рекуперативного теплообменника является энергетический коэфффициент, представляющий собой отношение количества переданной теплоты к затратам энергии на перемещение энергоносителей [5]. Рассмотрим задачу об оптимизации площади теплообменной поверхности рекуперативной горелки по принципу достижения максимума энергетического коэффрициента.

Задача оптимизации рассматривается для упрощенной постановки, когда задана и считается неизменной тепловая мощность горелки, выделяющаяся от сжигания топлива. То есть расходы топлива, воздуха и продуктов сгорания, приведенные к нормальным ффизическим условиям (н.ф.у.), также являются постоянными величинами, соответствующими тепловой мощности горелки. При заданных проходных сечениях каналов для воздуха и продуктов сгорания скорости воздуха и продуктов сгорания при н.фр.у. также неизменны. Коэффициент теплопередачи зависит не только от расхода топлива и режима горения, но и от конструктивных особенностей горелки. При решении поставленной задачи оптимизации конечные температуры воздуха и продуктов сгорания меняются не более чем на 10-15\%. Делается допущение о постоянстве коэффициента теплопередачи от продуктов сгорания к воздуху. Согласно расчетным оценкам, такой подход приведет к погрешности определения величины коэффрициента теплопередачи не более чем на $5 \%$. По этой причине делается предположение о постоянстве величины коэффрициента теплопередачи для каждого конкретного конструктива го- релки (высота каналов для прохода теплоносителей, параметры ребер и т.д.).

Методы исследования. При проведении численного исследования предполагается, что поверхность встроенного рекуператора горелки изменяется за счет изменения его длины [6]. Делается допущение, что напор вентилятора и разрежение эдуктора подстраиваются при изменении поверхности встроенного рекуператора (длины горелки) таким образом, чтобы обеспечить перемещение заданного расхода теплоносителей. Таким образом, требуемые напор и разрежение изменяются линейно относительно длины встроенного рекуператора.

Согласно $[7,8]$, теплообмен в теплообменнике рекуперативного типа удобно описать при помощи уравнения теплового баланса и выражения, связывающего температурные перепады на входе в теплообменник и на выходе из него:

- уравнение теплового баланса

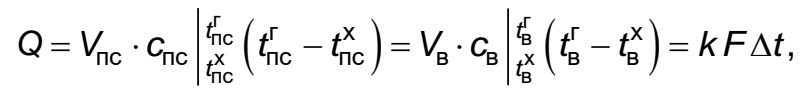

где $V_{\text {пс }}, V_{\text {в }}$ - объемные расходы продуктов сгорания и воздуха через рекуператор, $\mathrm{HM}^{3} / \mathrm{c}$; $t_{п \mathrm{x}}^{\mathrm{x}}, t_{\mathrm{B}}^{\mathrm{x}}, t_{п \mathrm{r}}^{\ulcorner}, t_{\mathrm{B}}^{\ulcorner}-$температуры продуктов сгорания на выходе, воздуха на входе, продуктов сгорания на входе, воздуха на выходе из рекуператора соответственно, ${ }^{\circ} \mathrm{C} ; \quad c_{\text {пс }} \mid \begin{aligned} & t_{\text {.r }}^{r} \\ & t_{\text {пc }}^{x}\end{aligned}$, $\left.c_{\mathrm{B}}\right|_{t_{\mathrm{B}}^{\mathrm{x}}} ^{t_{\mathrm{B}}^{\mathrm{r}}}$ - средние объемные теплоемкости продуктов сгорания и воздуха при температурах их нахождения в рекуператоре соот-

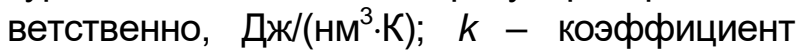

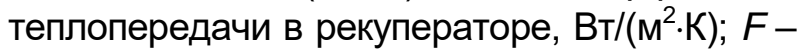


поверхность теплообмена рекуператора, м²; $\Delta t$ - среднелогарифмический температурный напор в рекуператоре, ${ }^{\circ} \mathrm{C}$;

- выражение, связывающее температурные перепады на входе и выходе из встроенного рекуператора:

$t_{\Pi \mathrm{C}}^{\mathrm{x}}-t_{\mathrm{B}}^{\mathrm{x}}=\left(t_{\mathrm{\Pi C}}^{\Gamma}-t_{\mathrm{B}}^{\ulcorner}\right) e^{-m k F}$,

где $m=\frac{1}{\left.V_{\text {пс }} \cdot c_{\text {пс }}\right|_{n_{\mathrm{n}}^{\mathrm{c}}} ^{t_{\text {तc }}^{r}}}-\frac{1}{\left.V_{\mathrm{B}} \cdot c_{\mathrm{B}}\right|_{t_{\mathrm{B}}^{\mathrm{x}}} ^{t_{\mathrm{B}}^{r}}}-$ параметр, характеризующий условия эксплуатации теплообменника при противотоке, определяемый в зависимости от водяных эквивалентов расходов теплоносителей, К.с/Дж.

Из совместного рассмотрения выражений (1) и (2) следует, что для определения искомых величин $\left(t_{п \mathrm{c}}^{\mathrm{x}}, t_{\mathrm{B}}^{\ulcorner}\right)$составляется система из двух уравнений:

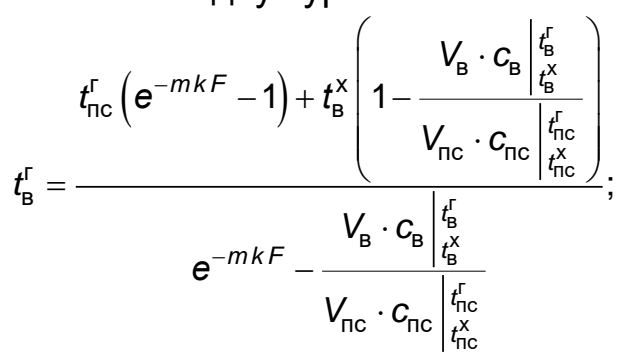

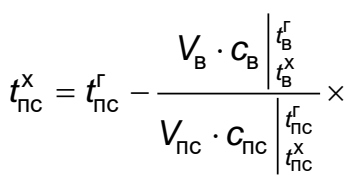

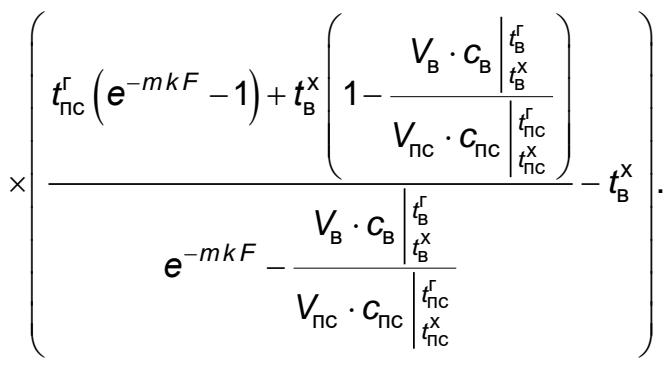

В литературе приведены более компактные способы записи выражений (3) и (4) с использованием функции ж противотока, которая табулирована в зависимости от соотношения водяных эквивалентов, коэффиициента теплопередачи и площади, значения которой приведены в таблицах. Приведенные в [9] уравнения изменения температуры горячего теплоносителя

$$
t_{1}^{\prime}-t_{1}^{\prime \prime}=\frac{1-e^{-\left(1-\frac{W_{1}}{W_{2}}\right) \frac{k F}{W_{1}}}}{1-\frac{W_{1}}{W_{2}} e^{-\left(1-\frac{W_{1}}{W_{2}}\right) \frac{k F}{W_{1}}}}=\left(t_{1}^{\prime}-t_{2}^{\prime}\right) \psi_{\text {прот }}\left(\frac{W_{1}}{W_{2}}, \frac{k F}{W_{1}}\right),
$$

а также температуры холодного теплоносителя $t_{2}^{\prime}-t_{2}^{\prime \prime}=\left(t_{1}^{\prime}-t_{2}^{\prime}\right) \frac{W_{1}}{W_{2}} \psi_{\text {прот }}\left(\frac{W_{1}}{W_{2}}, \frac{k F}{W_{1}}\right)$

вытекают из представленных выше выкладок для наглядной демонстрации влияния отдельных фракторов на исходную величину.

Установление значений искомых температур теплоносителей после встроенного рекуператора позволяет определить величину энергетического коэффициента:

$$
\begin{aligned}
& E_{э н}=\frac{V_{\mathrm{B}}\left(c_{\mathrm{B}}\left(t_{\mathrm{B}}^{\Gamma}\right) t_{\mathrm{B}}^{\Gamma}-c_{\mathrm{B}}\left(t_{\mathrm{B}}^{\mathrm{x}}\right) t_{\mathrm{B}}^{\mathrm{x}}\right)}{K_{\text {аэр }} F}= \\
& =\frac{V_{\text {пС }}\left(c_{\Pi \mathrm{CC}}\left(t_{\Pi \mathrm{r}}^{\Gamma}\right) t_{\Pi \mathrm{C}}^{\Gamma}-c_{\text {ПС }}\left(t_{\Pi \mathrm{x}}^{\mathrm{x}}\right) t_{\Pi \mathrm{C}}^{\mathrm{x}}\right)}{K_{\text {аэр }} F},
\end{aligned}
$$

где $K_{\text {аэр }}$ - коэфрфициент, численно равный итоговым затратам энергии на перемещение теплоносителей через встроенный рекуператор, приведенным к 1 м $^{2}$ гладкотрубной части поверхности рекуператора, Вт/м².

Данный коэффрициент учитывает потери на трение, местные сопротивления для каждого типа горелок и в общем определяется по следующей зависимости:

$K_{\text {аэр }}=\frac{\Delta P_{\text {пс }} V_{\text {пс }}+\Delta P_{\text {в }} V_{\text {в }}}{F}=$

$=V_{\text {пс }}\left(\lambda_{\text {пс }} \frac{l}{D_{\text {эк }}^{\text {Пс }}} \frac{\rho_{\text {пс }} w_{\text {пс }}^{2}}{2}+k_{\mathrm{MC}}^{\text {пс }} \frac{\rho_{\text {пс }} w_{\text {пс }}^{2}}{2}\right)+$

$+V_{\mathrm{B}}\left(\lambda_{\mathrm{B}} \frac{\mathrm{I}}{D_{Э \mathrm{~F}_{\mathrm{B}}^{\mathrm{B}}}^{\mathrm{B}}} \frac{\rho_{\mathrm{B}} w_{\mathrm{B}}^{2}}{2}+k_{\mathrm{MC}}^{\mathrm{B}} \frac{\rho_{\mathrm{B}} w_{\mathrm{B}}^{2}}{2}\right)$,

где $\Delta P_{\text {пс }}, \Delta P_{\text {в }}$ - сопротивление по стороне продуктов сгорания и воздуха соответственно, Па; $V_{\text {пс }}$ и $V_{\text {в }}$ - объемные расходы продуктов сгорания и воздуха соответственно, м ${ }^{3} /$ с; $\lambda_{\text {пс }}$ и $\lambda_{\text {в }}-$ коэфффициенты трения в каналах продуктов сгорания и воздуха соответственно; $D_{\text {экв }}^{\text {пс }}$ и $D_{\text {экв }}^{\text {В }}$ - эквивалентные диаметры каналов по стороне продуктов сгорания и воздуха соответственно, м; $\rho_{\text {пс }}$ и $\rho_{\text {в }}$ - плотность продуктов сгорания и воздуха соответственно, кг/. ${ }^{3}$; $w_{\text {пс }}$ и $w_{\text {в }}$ - скорость движения продуктов сгорания и воздуха соответственно, м/с; $k_{\mathrm{Mc}}^{\text {пс }}$ и $k_{\mathrm{Mc}}^{\mathrm{B}}-$ коэфрфициенты местных сопротивлений каналов продуктов сгорания и воздуха соответственно.

Для каждого конструктива горелки существует своя зависимость величины $K_{\text {аэр }}$ от текущей тепловой мощности горелки.

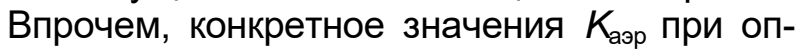
тимизации горелки для определенного значения тепловой мощности оказывает влия- 
ние на значения энергетического коэффиициента, но не меняет значения площади встроенного рекуператора, при котором достигается максимум целевой функции.

Пример определения зависимости энергетического коэффрициента от гладкотрубной части поверхности рекуператора произведен для исходных данных:

- мощность горелки, выделяющаяся от сжигания топлива - 500 кВт;

- топливо принято на 100 \% состоящим из метана;

- действительный удельный расход воздуха на сжигание топлива - 11,55 $\mathrm{m}^{3} / \mathrm{m}^{3}$;

- выход продуктов сгорания от сжигания топлива - $12,55 \mathrm{~m}^{3} / \mathrm{M}^{3}$;

- доля основного потока воздуха в горелке - $80 \%$.

Задача рассмотрена для двух значений коэфрицциента теплопередачи, отнесенных к гладкотрубной части поверхности рекуператора, - 150 и $200 \mathrm{BT} /\left(\mathrm{M}^{2} \cdot \mathrm{K}\right)$.

Результаты исследования. Результаты определения зависимости энергетического коэфффициента от площади гладкотрубной части поверхности встроенного рекуператора представлены на рис. 2.

Анализ полученных данных (рис. 2) показывает, что исследуемая зависимость имеет экстремальный характер - при определенном значении площади гладкотрубной части поверхности рекуператора достигается максимум энергетического коэффициента. Максимум является достаточно пологим, что снижет требования к точности определения и установления оптимального значения площади рекуператора.

Для определения величины $F_{\text {опт }}$ используется стандартный подход, согласно которому производная $d E_{э н} / d F$ приравнивается нулю и полученное уравнение разрешается относительно $F$. Полученный результат представляет собой искомую величину $F_{\text {опт. }}$

Задача определения $F_{\text {опт }}$ решалась для принятых в качестве примера исходных данных для значений коэффрициента теплопередачи, отнесенных к гладкотрубной части поверхности рекуператора, от 75 до $200 \mathrm{BT} /\left(\mathrm{M}^{2} \cdot \mathrm{K}\right)$. Решение итоговых уравнений производилось численными методами в среде стандартных инженерновычислительных пакетов.

Так, например, установлено, что для значений коэффрициента теплопередачи, отнесенных к гладкотрубной поверхности,
150 и $200 \mathrm{Bт} /\left(\mathrm{M}^{2} \cdot \mathrm{K}\right)$ оптимальные значения гладкотрубной части поверхности теплообмена составляют 1,07 и 0,8 м² соответственно.

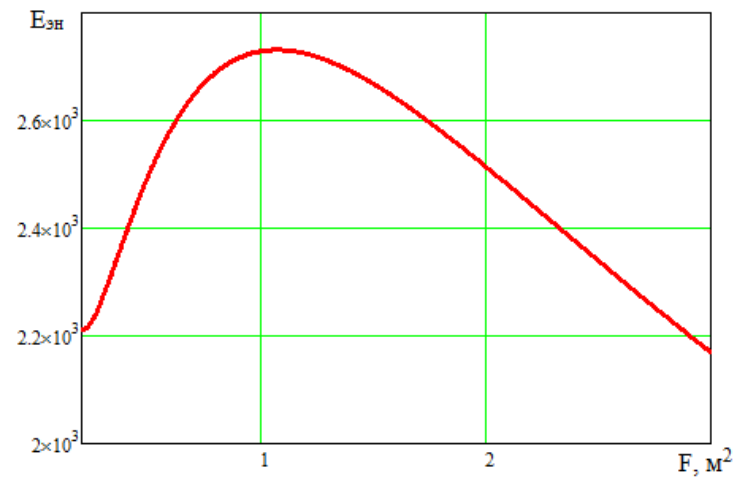

a)

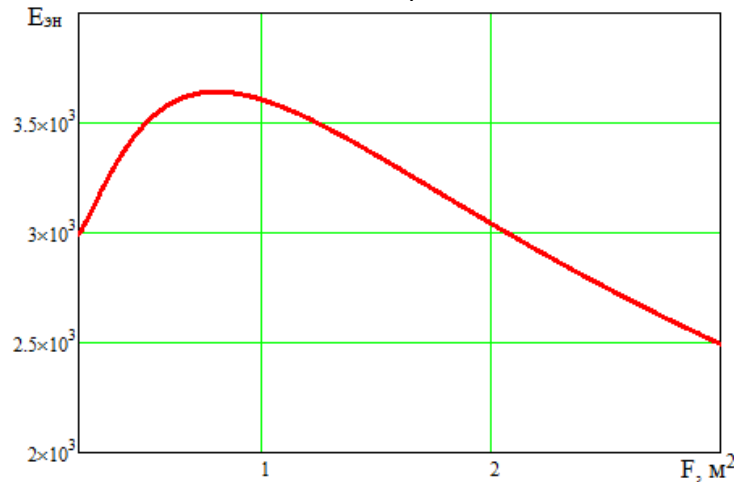

б)

Рис. 2. Зависимость энергетического коэффициента от площади гладкотрубной части поверхности встроенного рекуператора: а - для коэффициента теплопередачи $150 \mathrm{BT} /\left(\mathrm{M}^{2} \cdot \mathrm{K}\right) ;$ б для коэффициента теплопередачи $200 \mathrm{BT} /\left(\mathrm{M}^{2} \cdot \mathrm{K}\right)$

Полученные точки данных и аппроксимирующая кривая представлены на рис. 3. В качестве аппроксимирующего выражения использован полином пятой степени:

$F_{\text {опт }}(k)=5,137 \cdot 10^{-4}+0,132 k-2,399 \cdot 10^{-3} k^{2}+$

$+1,762 \cdot 10^{-5} k^{3}-5,891 \cdot 10^{-8} k^{4}+7,418 \cdot 10^{-11} k^{5}$.

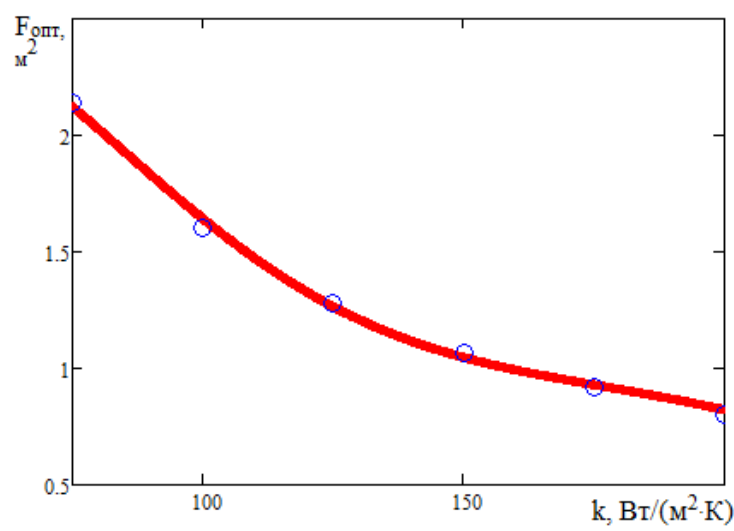

Рис. 3. Зависимость оптимальной величины площади поверхности гладкотрубной части рекуператора от величины коэффицциента теплопередачи 
Выводы. Использование разработанной методики проиллюстрировано на примере рекуперативной горелки с тепловой мощностью 500 кВт. Установлено, что для значений коэффициента теплопередачи, отнесенных к гладкотрубной поверхности, 150 и $200 \mathrm{BT} /\left(\mathrm{M}^{2} \cdot \mathrm{K}\right)$ оптимальные значения гладкотрубной части поверхности теплообмена составляют 1,07 и 0,8 м² соответственно. Для диапазона изменения коэффициента теплопередачи, отнесенного к гладкотрубной поверхности, от 75 до $200 \mathrm{BT} /\left(\mathrm{M}^{2} \cdot \mathrm{K}\right)$ зависимость оптимального значения гладкотрубной части поверхности теплообмена от коэфффициента теплопередачи описана в виде полинома пятой степени.

Исследование вида фрункциональной зависимости энергетического коэффрициента от поверхности теплообмена показало, что экстремум этой функции является достаточно пологим, что понижает требования к точности определения оптимального значения поверхности теплообмена.

В настоящее время отсутствует набор общепринятых методик теплотехнического расчета рекуперативных горелок. Разработанная методика важна для проектирования рекуперативных горелок, повышения их эффрективности и снижения стоимости их производства в отечественных условиях. Погрешность методики, связанная с допущением о постоянстве коэфффициента теплопередачи при изменении длины встроенного рекуператора, не превышает $5 \%$.

\section{Список литературы}

1. Топливосбережение при нагреве металла в печах машиностроительных предприятий / А.А. Шипко, И.А. Трусова, И.Н. Плющевский и др. // Литье и металлургия. - 2010. № 1(54)-2 (55). - С. 53-58.

2. Ядченко Д.С. Опыт использования рекуперативных горелочных устройств в печах периодического действия для термообработки заготовок в СПЦ-2 ОАО «БМЗ - управляющая компания холдинга "БМК"» // Литье и металлургия. - 2017. - № 4(89). - С. 32-36.

3. Вохмяков А.М., Казяев М.Д., Казяев Д.М. Исследование конвективного теплообмена в проходной печи, оснащенной скоростными горелками // Цветные металлы. - 2011. № 12. - С. 89-93.

4. Новая скоростная рекуперативная газовая горелка для прямого нагрева металла в промышленных печах / М.Р. Барташ, Г.М. Дру- жинин, Н.Б. Лошкарев и др. // Сталь. - 2010. № 3. - C. 125-127.

5. Калафати Д.Д., Попалов В.В. Оптимизация теплообменников по эффрективности теплообмена. - М.: Энергоатомиздат, 1986. $151 \mathrm{c}$.

6. Тепловой расчет рекуперативного теплообменного аппарата / В.В. Бухмиров, Д.В. Ракутина, Ю.С. Солнышкова, М.В. Пророкова. - Иваново, 2013. - 124 с.

7. Бирюков А.Б. Кравцов В.В., Косолюкин Д.А. Анализ мероприятий по повышению эфрфективности тепловой работы рекуператоров нагревательных печей // Металл и литье Украины. - 2011. - № 7. - С. 11-15.

8. Бирюков А.Б. Энергоэфффективность и качество тепловой обработки материалов в печах. - Донецк: Ноулидж, 2012. - 250 с.

9. Нащокин В.В. Техническая термодинамика и теплопередача: учеб. пособие для неэнергетических специальностей вузов. - М.: Высш. шк., 1975. - 496 с.

\section{References}

1. Shipko, A.A., Trusova, I.A., Plyushchevskiy, I.N., Korneev, S.V., Tolstoy, A.V. Toplivosberezhenie pri nagreve metalla $v$ pechakh mashinostroitel'nykh predpriyatiy [Fuel saving during the process of heating metal in furnaces of machinebuilding enterprises]. Lit'e $i$ metallurgiya, 2010, no. 1(54), 2 (55), pp. 53-58.

2. Yadchenko, D.S. Opyt ispol'zovaniya rekuperativnykh gorelochnykh ustroystv $v$ pechakh periodicheskogo deystviya dlya termoobrabotki zagotovok v SPTs-2 OAO «BMZ - upravlyayushchaya kompaniya kholdinga "BMK"» [The use of recuperative burner devices in batch furnaces for heat treatment of workpieces in SPTs-2 of BMZ Management Company of BMK Holding]. Lit'e $i$ metallurgiya, 2017, no. 4(89), pp. 32-36.

3. Vokhmyakov, A.M. Kazyaev, M.D., Kazyaev, D.M. Issledovanie konvektivnogo teploobmena $v$ prokhodnoy pechi, osnashchennoy skorostnymi gorelkami [Investigation of convective heat transfer in a continuous furnace equipped with high-speed burners]. Tsvetnye metally, 2011, no. 12, pp. 89-93.

4. Bartash, M.R., Druzhinin, G.M., Loshkarev, N.B., Popov, A.B., Khammatov, I.M. Novaya skorostnaya rekuperativnaya gazovaya gorelka dlya pryamogo nagreva metalla $v$ promyshlennykh pechakh [New high-speed recuperative gas burner for direct metal heating in industrial furnaces]. Stal', 2010, no. 3, pp. 125-127.

5. Kalafati, D.D., Popalov, V.V. Optimizatsiya teploobmennikov po effektivnosti teploobmena [Heat exchanger optimization for heat transfer efficiency]. Moscow: Energoatomizdat, 1986. 151 p.

6. Bukhmirov, V.V., Rakutina, D.V., Solnyshkova, Yu.S., Prorokova, M.V. Teplovoy raschet rekuperativnogo teploobmennogo apparata [Ther- 
mal calculation of recuperative heat exchanger]. Ivanovo, 2013. $124 \mathrm{p}$.

7. Biryukov, A.B. Kravtsov, V.V., Kosolyukin, D.A. Analiz meropriyatiy po povysheniyu effektivnosti teplovoy raboty rekuperatorov nagrevatel'nykh pechey [The analysis of measures to improve the efficiency of the heat operation of recuperators of heating furnaces]. Metall $i$ lit'e Ukrainy, 2011, no. 7, pp. 11-15.
8. Biryukov, A.B. Energoeffektivnost' i kachestvo teplovoy obrabotki materialov $v$ pechakh [Energy efficiency and the quality of heat treatment of materials in furnaces]. Donetsk: Noulidzh, 2012. $250 \mathrm{p}$.

9. Nashchokin, V.V. Tekhnicheskaya termodinamika $i$ teploperedacha [Technical thermodynamics and heat transfer]. Moscow: Vysshaya shkola, 1975. $496 \mathrm{p}$.

Бирюков Алексей Борисович,

ГОУВПО «Донецкий национальный технический университет», доктор технических наук, профессор, зав. кафедрой технической теплофизики, адрес: г. Донецк, ул. Кобозева, д. 15, ауд. 145a, e-mail: birukov.ttf@gmail.com Biryukov Aleksey Borisovich,

Donetsk National Technical University, Doctor of Engineering (Post-Doctoral Degree), Professor, Head of Engineering Thermophysics Department, address: Room 145a, 15 Kobozeva St., Donetsk, e-mail: birukov.ttf@gmail.com

Лебедев Александр Николаевич,

ГОУВПО «Донецкий национальный технический университет», кандидат технических наук, доцент кафедры промышленной теплоэнергетики, адрес: г. Донецк, ул. Кобозева, д. 15, ауд. 153, e-mail: lan@donntu.org Lebedev Alexander Nikolaevich,

Donetsk National Technical University, Candidate of Engineering (PhD), Associate Professor, Department of Industrial Heat Power Engineering, address: Room 153, 15 Kobozeva St., Donetsk, e-mail: lan@donntu.org

Гнитиёв Павел Александрович,

ГОУВПО «Донецкий национальный технический университет», кандидат технических наук, доцент, доцент кафедры технической теплофизики, адрес: г. Донецк, ул. Кобозева, д. 15, ауд. 145a, e-mail: gnitiev.pavel@gmail.com Gnitiev Pavel Alexandrovich,

Donetsk National Technical University, Candidate of Engineering (PhD), Associate Professor, Department of Engineering Thermophysics, address: Room 145a, 15 Kobozeva St., Donetsk, e-mail: gnitiev.pavel@gmail.com

Власов Ярослав Сергеевич,

ГОУВПО «Донецкий национальный технический университет», аспирант кафедры технической теплофизики, адрес: г. Донецк, ул. Кобозева, д. 15, ауд. 145a, e-mail: yaroslav.vlasov.1994@mail.ru Vlasov Yaroslav Sergeevich,

Donetsk National Technical University, Postgraduate student, Technical Thermophysics Department, address: Room 145a, 15 Kobozeva St., Donetsk, e-mail: yaroslav.vlasov.1994@mail.ru

\title{
ИССЛЕДОВАНИЕ РЕЖИМОВ РАБОТЫ ГАЗОВОЙ ТУРБИНЫ ГТЭ-110 ПО УСЛОВИЯМ ОГРАНИЧЕНИЯ ВЫБРОСОВ ОКСИДОВ АЗОТА НА ПАРОГАЗОВЫХ ЭНЕРГОБЛОКАХ
}

\author{
И.К. МУРАВЬЕВ, А.В. КОРОВКИН, Р.А. ШИТОВ \\ ФГБОУВО «Ивановский государственный энергетический университет имени В.И. Ленина», \\ г. Иваново, Российская Федерация \\ E-mail:kafsu@ispu.ru, a.korovkin37@gmail.com, pomas@inbox.ru
}

\section{Авторское резюме}

Состояние вопроса. Газовые турбины активно используются в составе парогазовых энергоблоков, оказывая меньшее влияние на окружающую среду, чем установки, работающие на других видах топлива. Однако их выбросы содержат вредные углеродистые соединения и окислы азота. В существующей литературе рассматривается влияние выбросов при изменениях коэфффициента избытка воздуха. При этом не уделяется внимания влиянию других режимных параметров и технологических ограничений, связанных с безопасной эксплуатацией совместно работающего оборудования парогазовых энергоблоков, а также не дана оценка влияния климатических фракторов на экологические показатели. В свя- 